\title{
Possibilities of increasing the in-service durability of bearing shells used in rolling mills
}

\author{
Adrian Constantin Tanasie ${ }^{\mathbf{1}}$, \\ ${ }^{\text {I}}$ WEST.RO SUPPORT SRL, Timisoara, Romania \\ E-mail:aditanasie74@gmail.com
}

\begin{abstract}
This paper shows how to use the spent cells from the geoning of laminates. Building stages of the molding mix in the shells are preset which is extending their period of operation and then achievement of significant economic savings.

Keywords: Wear, reconditioning, bearings, bearing shell, composition.
\end{abstract}

\section{Introduction}

During operation, the bearing shells become worn by various factors, i.e. they change the properties of the surface layers, which often makes them unusable.

In general, friction has a harmful effect on any part due to its effects: Changing the structure and chemical composition of the surface coats in case of overheating, loss of material, deterioration in the quality of the lubricant used due to the deposition of abrasive particles, etc.

The wear of the machine parts and components depends on the quality of the processed surfaces, the materials used and the lubricants, the specific speed and pressure of the workpieces under friction, the clearance between the parts and the operating conditions.

A major concern today is the reconditioning of parts.

The purpose of reconditioning technically worn parts is to remove defects and restore the initial adjustments to the assemblies, to ensure high reliability and sustainability, and to save money from a reconditioning point of view.

The cost of reconditioned parts and sub-assemblies shall be determined taking into account all expenses incurred in reconditioning them.

The durability of reconditioned parts depends on a variety of factors including: The methods and procedures for reconditioning chosen, the quality of reconditioning operations and the materials used for this purpose, all of which play a key role.

Quality is required as the manufacturer's standard, so reconditioning is done according to the same specifications as for new parts. Reconditioned parts are subject to the same safety tests as the series. Their quality is derived from the fact that due care and precision, the reconditioning procedure is restored to its original characteristics and guaranteed to function perfectly over time.

Therefore, if the reconditioned part has the original quality and a price much lower than the new one, this is the solution that always wants the end user, the best value for money.

\section{Role of bearing shells in the bearings of metalworking machinery}

The machines of the rolling sections are subject to considerable strain at all times. These can be: Mechanical (shock, wear, fatigue), thermal, chemical (water, steam, acids, etc.) and associated. 
Given the difficult conditions in which the bearings of metalworking machinery, characterized by high pressures and temperatures, their maintenance, operation and repair are highly responsible operations and immediate consequences for the proper functioning of the rolling mill as a whole.

The bearing shell is intended to support and guide shafts and axles in their rotation movement under operating loads. They are sleeves in one or two halves. It is generally done in bronze and sometimes for smaller cast iron loads. The bearing shells can be coated with a bearing shell composition molded directly onto the inner face of the bearing shells.

Our example will be a statically molded YSn83 steel liner. The bearing shell will be of large size and endure considerable loads both axially and radially. The inside diameter is $400 \mathrm{~mm}$, the outside $500 \mathrm{~mm}$ and the length $500 \mathrm{~mm}$.

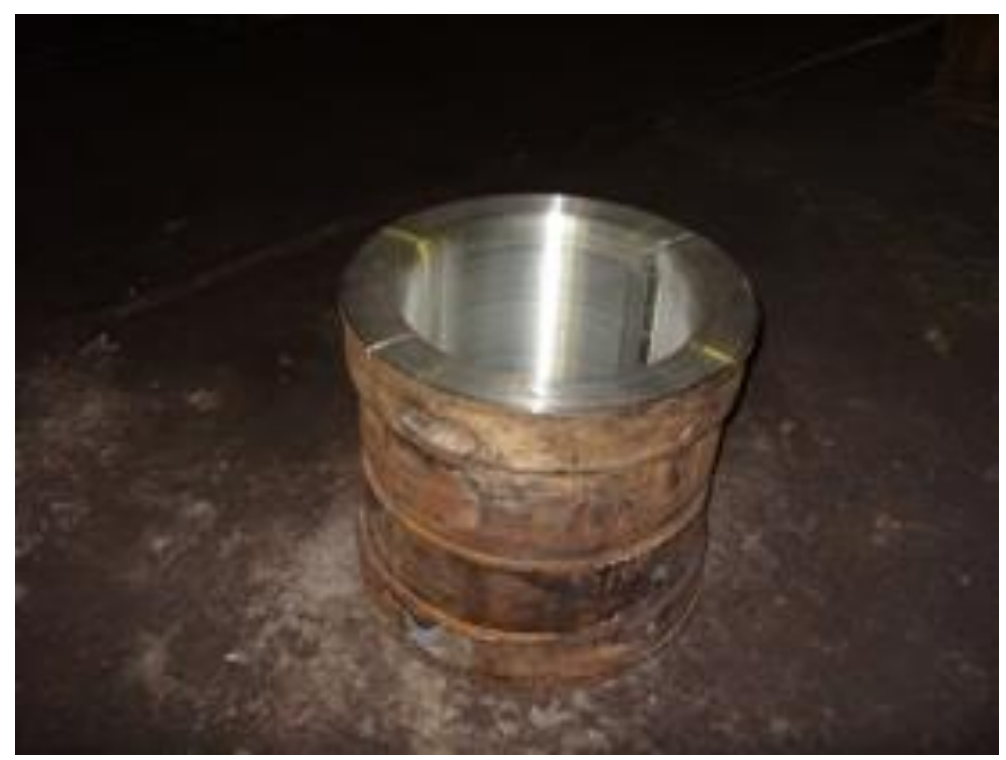

Figure 1. Solid molded shell with liner

The bearing shell is subjected to heavy loads that are transmitted from the rolling mills and are mounted in the gear case. It is precisely because of this that the slip bearings must meet the following requirements simultaneously:

- to withstand loads (radially or axially);

- permit relative translation or rotation movements;

- ensure the relative position of the contact surfaces;

- ensure low friction and wear.

\section{Anti-friction material}

The kinematic coupling made up of the Journal and bearing shell only works correctly if the equipment is correctly chosen. Anti-friction material forms a liner on a durable material.

The most commonly used bearing materials used to make these bearing shells are: tanning substances (based on tin, lead, aluminum), sintered metal materials: powders of iron, copper, tin, lead, graphite, pressed at high temperatures; bimetal materials; non-metallic materials : plastics (textiles, polyamides), ceramic materials, graphite.

The anti-friction material shall have the following properties:

- have a melting temperature higher than the bearing operating temperature;

- have high thermal conductivity;

- have a coefficient of expansion close to that of the supporting material;

- be cheap. 
Even if the bearing shell meets all these properties the wear process cannot be avoided. This process appears as a complex phenomenon, due to various causes and determined by a large number of factors and conditions (mechanical properties, geometric micro or macro characteristics of the surfaces, functional parameters, lubrication quality, lubricants used).

In order to reduce bearing friction effects, lubricants are used which should separate the friction surface as completely as possible. Lubricants are also used to remove heat from friction and to protect surfaces in contact with corrosion.

Wear is a progressive destructive process of a physico-chemical nature, which has the main effect of causing wear.

Figures 2 and 3 show breaks caused by overloads and gaps left as a result of pouring. At the same time, the grit caused by impurities that have reached between the Journal and the bearing shell probably by means of lubrication oil is visible.

Overloads cause stress on machinery organs that can exceed the limits of resistance. Even if the working requirements (e.g., shaving regimes) are followed precisely, overloads may occur when the machine, machine, or installation has an advanced degree of wear. In this situation the friction increases so much that the permissible load is exceeded. Overbursts, by their sudden action, increase the beats, speed up the wear and can even lead to the destruction of some of the machine's components.

With these overloads, the friction between the bearing shell and the Journal increases, the torque temperature increases and the anti-friction material can melt, thus causing the bearing shell to become stuck. The bearing shell can be removed, the anti-friction material on the base metal part is melted and the casting technology is restored.

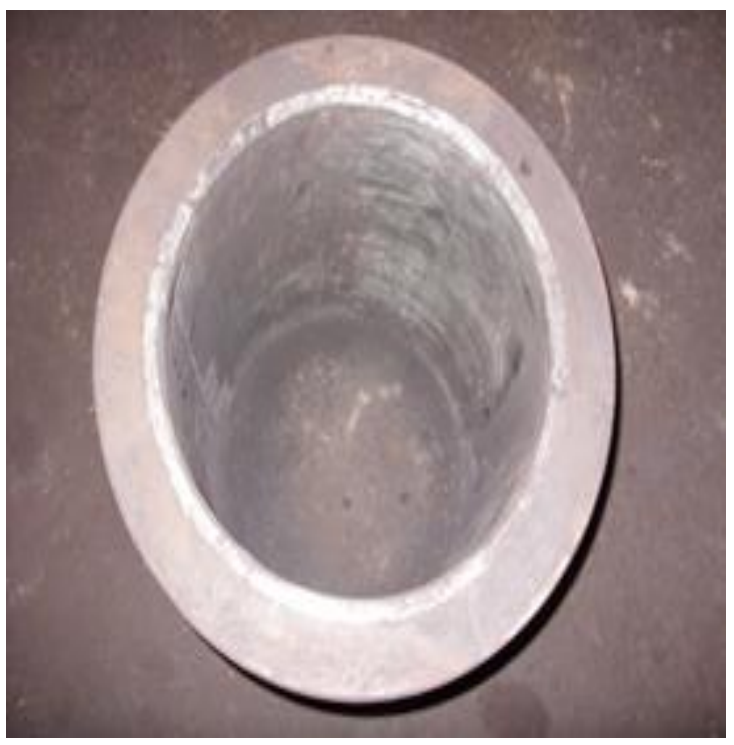

Figure 2 Worn bearing shell with faults

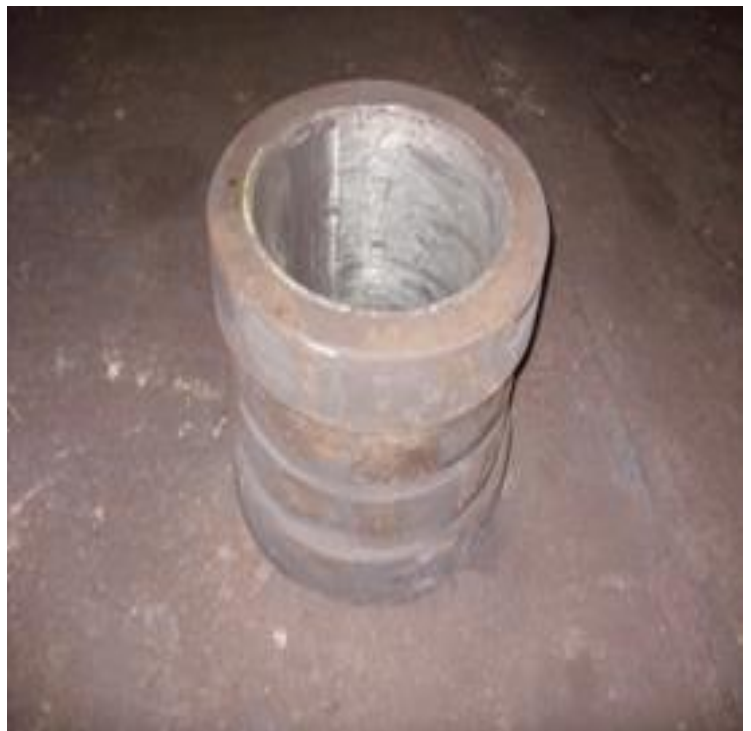

Figure 3 Worn bearing shell with grit molding

\section{Preparation of the shell and actual casting}

For static casting of composition into the bearing shells, the following steps are carried out:

- preparation of the surface of the bearing shell by mechanical and chemical means;

- bearing shell tin;

- melting the composition;

- the fitting and fitting of bearing shells;

- casting of composition. 


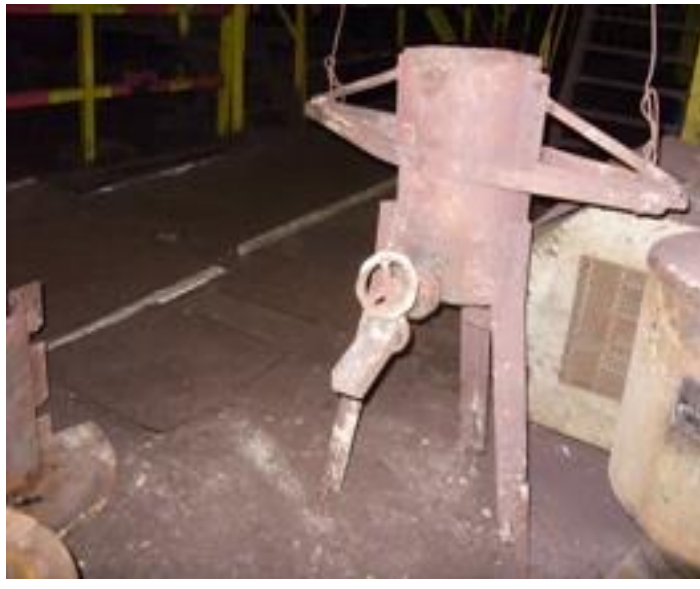

Figure 4 The molding pot

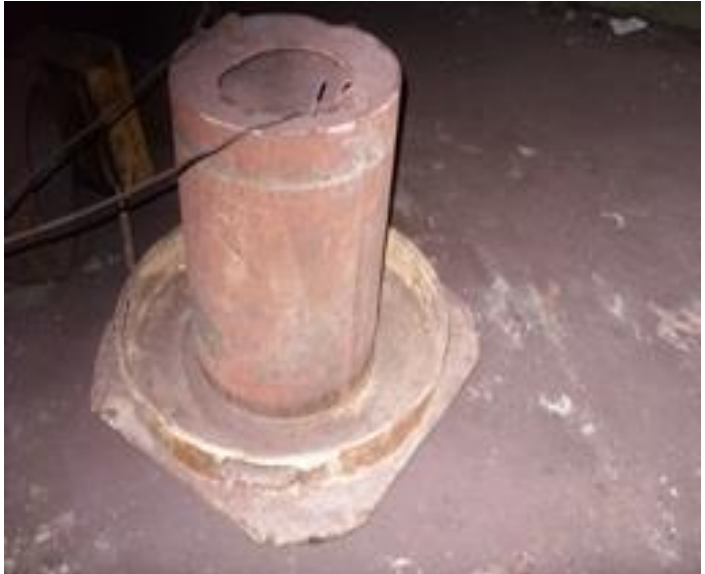

Figure 5 Shape of molding

The preparation of the bearing shell surface consists of:

- cleaning with a steel wire brush, abrasive paper, of the channels in the form of a rendertail which are intended to prevent the composition from falling;

- degreasing of the bearing shells for $1 / 2$ minutes with an aqueous solution of caustic soda (ca.130g soda per 1 liter water) heated to boiling;

- washing the flush bearing shell in hot water and then in cold water to remove all the remains of the soda from its surface;

- placing the bearing shell for 2-5 minutes in an aqueous solution with $50 \%$ hydrochloric acid,

- washing the shell in an aqueous solution of $10 \%$ hot caustic soda to remove leftovers; Bearing shell mashing is carried out either by steeping or rubbing.

For diving tin, cover the outer surface of the shell with a protective coating, made of $0,5 \mathrm{~L}$ soluble glass and 4 glasses of chalk powder per $1 \mathrm{~L}$ water.

After drying the insulating layer, the molten alloy bearing shell is immersed for approximately 2 minutes. After removal from the molten alloy bath, shake and wipe with chalks or an asbestos brush.

For tinting by friction, place the bearing shell on a support, moisten with flux the surface to be tinned and heat the bearing shell. Then a tin-alloy verb approached the surface of the shell and with a sweep the molten alloy layer spread evenly. At the end of the operation, press typirige and wipe with clean limes or an asbestos brush.

Melt the composition in crucibles of cast iron, steel or graphite heated under a forja fire. Place the mixture in the crucible when it is heated thoroughly. The surface of the batter is covered with a layer of 20 to $30 \mathrm{~mm}$ of dry charcoal to avoid oxidation. The temperature of the composition must be above the melting temperature by a minimum of $600 \mathrm{C}$ so that at the time of pouring it is as fluid as possible and does not immediately solidify in the bearing shell.

The bearing shells are fitted and positioned as follows:

- the lower part of the shape which is along with the core of the shape shall be placed on a level surface;

- an asbestos film shall be inserted in the shell separation planes to eliminate all the slots;

- the bearing shell is positioned so that the center of the shape coincides with the center of the bearing shell;

- tighten the bearing shells with a band;

- with a paste of $65 \%$ clay, $17 \%$ kitchen salt, $18 \%$ water is clogged all the louvers outside. 
Pouring of the composition is carried out with spoons which must be of sufficient capacity for the pouring to be carried out without interruption. Before the spoon is immersed in the molten crucible, release the coal and the slag-containing film formed. The composition is poured into a thick, short stream to avoid the formation of souls in the solidified composition.

Another way to pour the composition is directly from the casting pot, which is equipped with a cep on the bottom which allows the composition flow to be started and stopped, thus avoiding the formation of souls.

For pouring the composition into a single bearing shell, a template shall be used, the bending radius of which shall be large enough that a space equal to the required thickness of the composition layer remains between the template and the bearing shell.

The bearing shells are checked visually and by means of a sound test after complete cooling.

The working surface of the bearing shell must be smooth, silver-colored, free of yellow or black spots, and the sound produced by striking the suspended shell with a hammer must be clear.

\section{Conclusions}

Reconditioning the bearing shells has certain advantages related to:

-savings in raw materials and energy by extending their service life;

-the possibility of modifying the chemical composition and microstructure of the deposited layer, thereby increasing the operational performance;

-re-use of the base metal part of the bearing shell requiring expensive manufacturing technology, reduction of production costs.

The effective operation of the loaded parts is dependant on knowledge and proper use of the specific characteristics of all the components used to obtain the base metal assembly and the addition.

Recovering and reconditioning parts will continue to be actions of great importance to any country regardless of the level of prosperity that characterizes its economy.

Recovering and reconditioning means saving important social work, means working permanently to protect internal material and energy resources, helping to eliminate the dependence of economic development on the fluctuations in world trade.

\section{References}

[1] Berinde, V., recovering, reconditioning and re-using parts, Technical Publishing, Bucharest, 1986.

[2] Mitroi, C., and so on, technology for refurbishing used pieces, Technical Publishing, Bucharest, 1988.

[3] Radulescu, C., Gutu, M., Derlogea, S., laminating machinery, Technical Publishing, Bucharest, 1979.

[4] Radut, N., s., rebuild parts, Military Publishing, Bucharest, 1983.

[5] Heyne, P., economy way of thinking, Didactica and pedagogical Publishing House, Bucharest, 1991. 